\title{
Piezo1 induced apoptosis of type II pneumocytes during ARDS
}

\author{
Guo-Peng Liang ${ }^{1 \dagger}$, Jing Xu ${ }^{1 \dagger}$, Li-li Cao ${ }^{2}$, Yi-Hua Zeng ${ }^{3}$, Bai-Xu Chen ${ }^{1}$, Jing Yang ${ }^{1}$, Zhong-Wei Zhang ${ }^{1 * \dagger}$ and \\ Yan Kang ${ }^{1 * \dagger}$
}

\begin{abstract}
Objective: The mechanisms of lung injury in acute respiratory distress syndrome (ARDS) are not well understood.Piezo 1 was recently identified as a mechanotransduction protein. The present study found the expression of Piezo1 in type II pneumocytes and investigated its role in mediating ARDS-related lung injury.

Methods: Sprague-Dawley rats were used to establish an ARDS model, the expression of Piezo1,lung injuries, apoptosis as well as calcium influx were assessed.

Results: Piezo1 was expressed in type II pneumocytes as shown by immunofluorescence staining and expression was increased in the ARDS model. Knockdown of Piezo1 reduced apoptosis which was related to the elevation of Bcl-2.Calcium influx played a vital role in Piezo1-induced apoptosis.

Conclusion: Piezo1 was expressed in type II pneumocytes. Mechanical stretch of alveoli during ARDS induced activation of the Piezo1 channel, which resulted in calcium influx. The increased intracellular Ca2+ induced the apoptosis of type II pneumocytes, which may be related to the Bcl-2 pathway.
\end{abstract}

Keywords: Acute respiratory distress syndrome, Lung injury;Piezo1;apoptosis;Ca2+

\section{Background}

Acute respiratory distress syndrome (ARDS) is a common and life-threatening respiratory disorder of inflammatory lung injury responsible for $10 \%$ of all ICU admissions and a has a mortality rate of nearly $40 \%$ [1]. It is a type of acute diffuse, inflammatory lung injury, leading to increased pulmonary vascular permeability, increased lung weight, and loss of aerated lung tissue [2]. Recent evidence suggested that the main pathogenesis of ARDS included cytokine imbalance oxidative stress, and apoptosis of alveolar epithelial cells [3].However, the mechanisms of ARDS-related lung injury have not been clarified well.

Piezo1 was first detected in murine neuroblastoma cells and was recently identified as a component of mechanically-activated cation channels [4]. which were

\footnotetext{
* Correspondence: 716461751@qq.com; kangyan@scu.edu.cn

${ }^{\dagger}$ Guo-Peng Liang and Jing Xu contributed equally to this work and should be considered co-first authors.

†Yan Kang and Zhong-Wei Zhang contributed equally to this work. 'Department of Critical Care Medicine, West China School of Medicine and West China Hospital, Sichuan University, Chengdu 610041, China Full list of author information is available at the end of the article
}

inherently mechanosensitive without relying on alternate cellular components for sensing mechanical stimuli [5]. Application of the force on cells caused calcium to rapidly enter cells through Piezo1 channels [6-8], which played an important role in sensing mechanical stretch. Piezo1 expressed in platelets, this contributed to $\mathrm{Ca} 2+$ entry and thrombus formation under arterial shear [9]. Piezo1 was found to be expressed in endothelial cells of developing blood vessels, and knockout of Piezo1 was embryonically lethal with defects in vascular remodeling [10]. In addition,Piezo1 expressed in arterial smooth muscle cells was able to sense the fluid shear stress exerted by blood flow and affect arterial structure(diameter and wall thickness) by releasing ATP and regulating of NO formation to control vascular tone and blood pressure [11-13] . Piezo1 also senses extension of the bladder urothelium [14], intraluminal pressure changes and urine flow sensing [15]. These studies showed that Piezo1 is capable of detecting various kinds of mechanical stress and is involved in biological processes and maintenance of normal structures of human organs. 
During respiration, alveoli and airways are stretched repetitively, and are constantly under mechanical stress generated by air and the surrounding tissues. Respiratory disorders tend to enhance mechanical stretch by a series of pathophysiological mechanisms, that lead to lung injury. Mechanical ventilation is another important factor that may increase lung injury due to tidal hyperinflation [16, 17], and cyclic recruitment/derecruitment [18]. Given that Piezo1 is a mechanosensitive ion channel with the ability to sense mechanical stretch, we assumed that Piezo1 was also expressed in the lung and may be a potential mechanism of lung injury. The mRNA expression profile indicated that Piezol may be expressed in the lung [4]. However, to the best of our knowledge, no studies have identified the physiological functions of Piezo1 in lung injury.

Therefore, the present study was designed to investigate the expression and functions of Piezo1 in the lung and study the potential mechanisms of Piezo1 in ARDSrelated lung injury. We attempted to find a new target for prevention and treatment of ARDS.

\section{Materials and methods}

\section{Animals and experimental design}

The protocol was approved by the Institutional Animal Care and Treatment Committee of Sichuan University (Chengdu, P.R. China). Health adult Sprague-Dawley (SD) rats weighing $250 \pm 10 \mathrm{~g}$ (Experimental Animal Center of Academy of Military Medical Sciences of the People's Liberation Army, China) were housed in cages with free access to water and food and divided randomly into four groups: 1) control group, 2)ARDS group, 3)ARDS+ high tidal volume(HV)group and 4)ARDS+ low tidal volume(LV) group,(five rats per group). Control rats were injected with $0.1 \mathrm{~mL} / \mathrm{kg}$ body weight physiological saline while those in experimental groups were given $0.1 \mathrm{~mL} / \mathrm{kg}$ body weight oleic acid by intravenous injection and the rats were spun around 10 times to establish ARDS model which was then confirmed by assessing partial pressure of oxygen in the arterial blood after $3 \mathrm{~h}$. ARDS+HV and ARDS+LV rats were ventilated with a tidal volume of 10 $\mathrm{mL} / \mathrm{kg}$ and $6 \mathrm{~mL} / \mathrm{kg}$ respectively,with positive endexpiratory pressure (PEEP) $3 \mathrm{cmH} 2 \mathrm{O}$, and respiratory frequency of $25 / \mathrm{min}$ for $4 \mathrm{~h}$. Next, rats in all groups were anaesthetized, and lung sections were collected.

\section{Assessment of lung injury}

Blood(50ul) was collected from aorta abdominalis and partial pressure of oxygen and carbon dioxide $(\mathrm{PaO} 2$,and $\mathrm{PaCO}$,respectively)was measured by blood gas analyzer (Abbott i-STAT). Oxygenation index was calculated as $\mathrm{PaO} 2 / \mathrm{FiO} 2$, and lung morphology was observed by hematoxylin and eosin(HE)staining of left lung tissue. The wet/dry ratio (W/D ratio) was calculated to reflect the severity of pulmonary edema. Wet weights of right lower lungs were measured upon collection of lung tissue, and then lungs were incubated in an oven $\left(60^{\circ} \mathrm{C}\right.$ for $\left.72 \mathrm{~h}\right)$ to measure dry weight. The levels of serum TNF- $\alpha$ and IL-1 $\beta$ were measured by ELISA assay(MD6692,MD6693).

\section{Cell culture and transfection}

Human lung epithelial cell line A549 was seeded on 10 $\mathrm{cm}$ dishes in DMEM, and cultured at $37^{\circ} \mathrm{C}$ and $5 \% \mathrm{CO} 2$. Cells were transiently transfected with either Piezo1 siRNA or control siRNA using Lipofectamine 2000(Invitrogen, Carlsbad, CA, USA;11,668,019),the sequence of siRNA is provided in Additional file 1: Figure S2-A.

\section{RNA isolation, real-time q-PCR, and western blots}

After $24-48 \mathrm{~h}$ post transfection, total RNA was extracted using Trizol(Invitrigen)following the manufacturers' instructions. Complementary DNAs (cDNAs) were synthesized using the HiScript ${ }^{\circ}$ II One Step RT-PCR Kit (Vazyme Biotech, Nanjing, China)and amplified using specific primers for Piezo1.qPCR was performed with a LightCycler 480 system(Roche, Basel, Switzerland). GAPDH was used as a reference gene for relative quantification of Piezo1 gene expression. The primer sequences are as follows:

Piezo1-F 5'-GCTGTACCTGCCTGATTTCTTCC-3' .

Piezo1-R 5'-CATGCGCTGCCACTCCTCT-3';

GAPDH-F 5'-CACATGGCCTCCAAGGAGTAA-3'; and.

GAPDH-R 5'-TGAGGGTCTCTCTCTTCCTCTTGT$3^{\prime}$.

Western blots were performed as previously described [19]. Antibodies included Piezo1 (Santa Cruz Biotechnology, Dallas, TX, USA; sc-164,319), dilution ratio 1:1000),Bcl2(Abgent, San Diego, CA, USA, dilution ratio 1:1000),caspase 3 + cleaved caspase3(Cell Signaling Technology, Danvers, MA, USA; $9664 \mathrm{t}$, dilution ratio 1:2000) and GAPDH (Abcam, Cambridge, UK; Ab8245; dilution ratio 1:1000).

\section{Isolation of lung type II pneumocytes}

Type II pneumocytes were isolated from SD rats by a modified method as previously described [20]. Briefly, the cannula was inserted into the pulmonary artery through the right ventricular and the right lung was lavaged with normal saline supplemented with heparin for 2-3 min, then the heart and lungs were quickly severed together with the trachea and taken out from the body. Lavage was performed through trachea cannula using normal saline $(5 \mathrm{~mL} \times 8)$, then $2 \mathrm{ml}$ PBS containing $0.1 \%$ collagenase and $0.125 \%$ trypsin was infused into the right lung and the lung was incubated the lung in $5 \% \mathrm{CO}_{2}$ at $37^{\circ} \mathrm{C}$ for $25 \mathrm{~min}$. The lung was cut into small pieces and FBS was used to inactivate trypsin, then the lung was transferred into $20 \mathrm{~mL}$ PBS containing 1 $\mathrm{mL}$ DNAse I, and incubated in a $37^{\circ} \mathrm{C}$ water bath for $5 \mathrm{~min}$. Lung single suspension were filtered through 60,150 and 
280 mesh filters in turn and the supernatant was discarded after centrifugation. The pellet was resuspended in DMEM media and seeded in $10 \mathrm{~cm}$ dish for $1 \mathrm{~h}$. Then, the unadhered cells were collected and transferred into another dish. Finally, the cells were identified by immunofluorescence staining of pulmonary surfactant protein $\mathrm{C}(\mathrm{SP}-\mathrm{C})$,the marker of type II pneumocytes.

\section{Immunofluorescence and immunohistochemistry}

Expression of Piezo1 was first examined by immunohistochemical staining in lung tissues and then examined by immunofluorescence staining. For immunohistochemistry, paraffin-embedded and dewaxed lung sections were exposed to citrate buffer for antigen unmasking, then blocked for $30 \mathrm{~min}$ using BSA. Sections were incubated overnight at $4{ }^{\circ} \mathrm{C}$ with Piezo1 and SP-C primary antibodies, then incubated with horseradish peroxidase (HRP) for $30 \mathrm{~min}$ the next day. For immunofluorescence, lung sections were prepared in the same manner but incubated with secondary antibodies at $37^{\circ} \mathrm{C}$ for $30 \mathrm{~min}$ the next day. Then, sections were stained with DAPI (Beyotime,Shanghai,China;C1002) and the images were captured using a fluorescence microscope(Leica, Wetzlar, Germany;DM500) .

\section{Apoptosis assay}

For primary cells derived from SD rats, single cell suspensions were prepared as mentioned in the "Isolation of lung type II pneumocytes cells" section. For cell lines, A549 cells were digested by trypsin without EDTA. The cell concentration was adjusted to $3 \times 10[5] / \mathrm{mL}$ and stained with a 100ul binding solution containing $5 \mathrm{ul}$ annexin V-fluorescein isothiocyanate and 5ul PI solution in a 100ul binding solution. Apoptosis was assayed using a Novo Cyte flow cytometry (Acea Biosciences, San Diego, CA, USA) .

\section{Mechanical stretch and calcium imaging}

BioFlex culture plates were coated with type I collagen in $4{ }^{\circ} \mathrm{C}$ for $16-24 \mathrm{~h}$. A549 $(5 \times 10$ [5])cells were then seeded on the plates for $24 \mathrm{~h}$, and fresh serum-free medium was used before applying mechanical stretch. FX-4000 (Flexercell Corporation, Burlington, NC, USA) was used to produce mechanical stretch on cells with a square wave.The mechanical force intensity was set up as $15 \%$,frequency $0.5 \mathrm{HZ}$, and lasted for $4 \mathrm{~h}$. The control group was subjected to the same procedure but without stretch stimulation. After the experiment, cells were collected and washed twice with PBS and resuspended in $1 \mathrm{~mL}$ PBS, and then 500ul of $10 \mu \mathrm{M}$ calcium probes (Fluo-3/AM, Beyotime; S1056) was added into cell suspensions and incubated in the dark at $37^{\circ} \mathrm{C}$ for $30 \mathrm{~min}$. Confocal imaging (TCS SP8,Leica,German) was performed to detect $\mathrm{Ca} 2+$.Fluo-3 AM fluorescence signals were measured at $488 \mathrm{~nm}$ excitation wavelength and $528 \mathrm{~nm}$ emission wavelength. We chose eight visual fields and calculated the fluorescence intensity and cell counts with Image Pro Plus 6.0.The calcium signal was calculated as total fluorescence intensity/ cell counts.

\section{Statistical analysis}

The data were analyzed using SPSS 19.0 software and expressed as mean \pm standard error. The results in different groups were compared by one-way ANOVA and post hoc Tukey was used for multiple comparisons. $P<$ 0.05 was considered statistically significant.

\section{Results}

The expression of Piezo1 in type II pneumocytes

From immunohistochemical staining, Piezol was found to be expressed in the rat lung, mainly located around the alveoli(Fig. 1a). As type II pneumocytes are one of the components of alveolar epithelia and have a crucial role in maintaining regular alveolar morphology, we speculated whether Piezo1 was expressed in type II pneumocytes. Surfactant protein C (SP-C) was used to specifically label type II pneumocytes by immunofluorescence staining. Double-staining showed that Piezo1-positive cells were mostly positive for SP-C(Fig. 1b), confirming that Piezo1 was predominantly expressed in type II pneumocytes.

Piezo1 induced apoptosis of type II pneumocytes in ARDS To investigate the role of Piezo1 in ARDS, the oleic acidinduced ARDS model was established in rats and confirmed by assessing oxygenation index, wet/dry ratio, $\mathrm{HE}$ staining(Fig. 2a) and other indicators(Additional file 1: Figure S1A) We next compared Piezo1 expression between control and ARDS rats, and found that Piezo1 was significantly increased in the ARDS group(Fig. 2b), indicating that Piezo1 may play a role in ARDS progression. As Piezo1 was

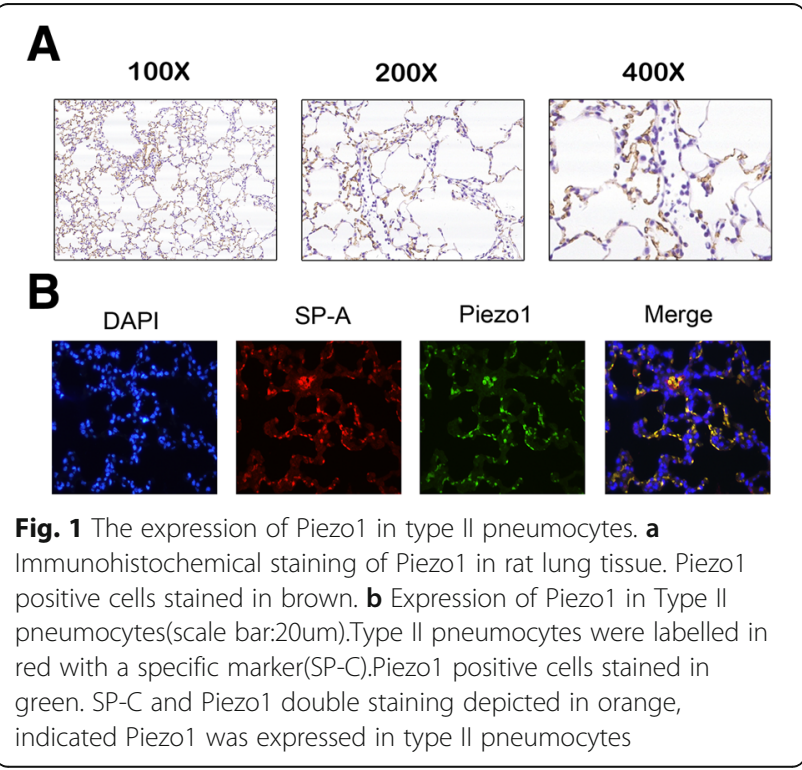




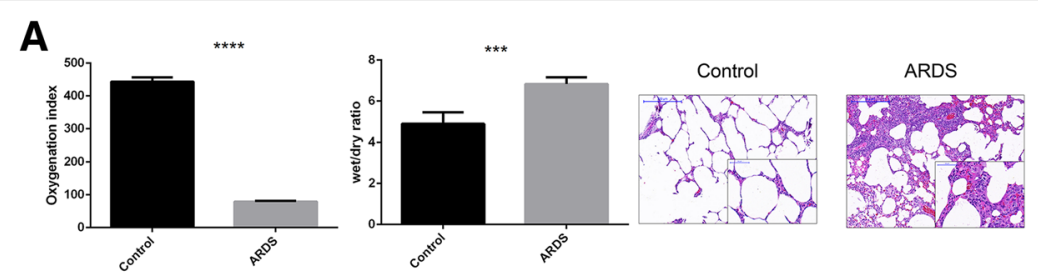

B

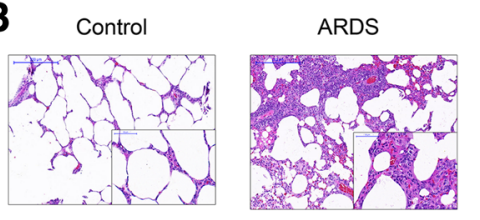

C
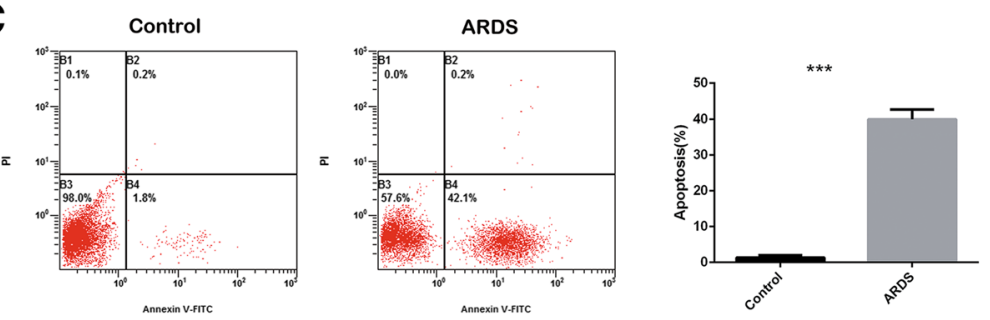

D
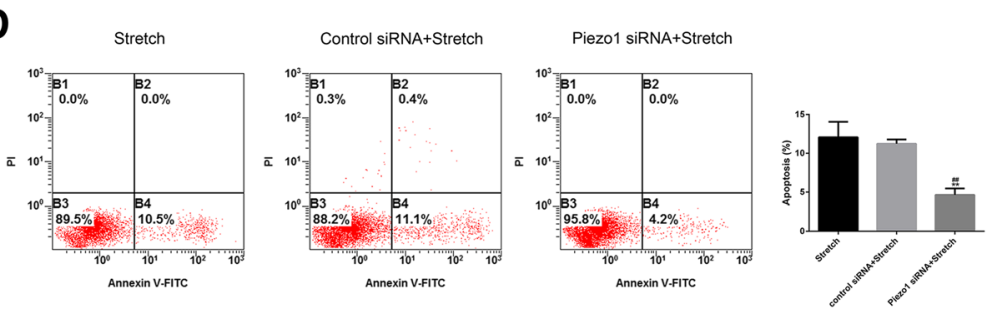

E

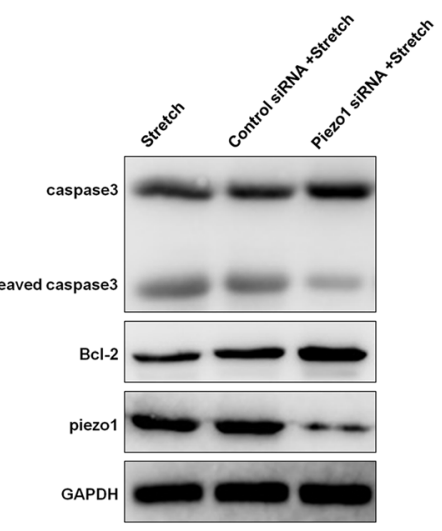

F
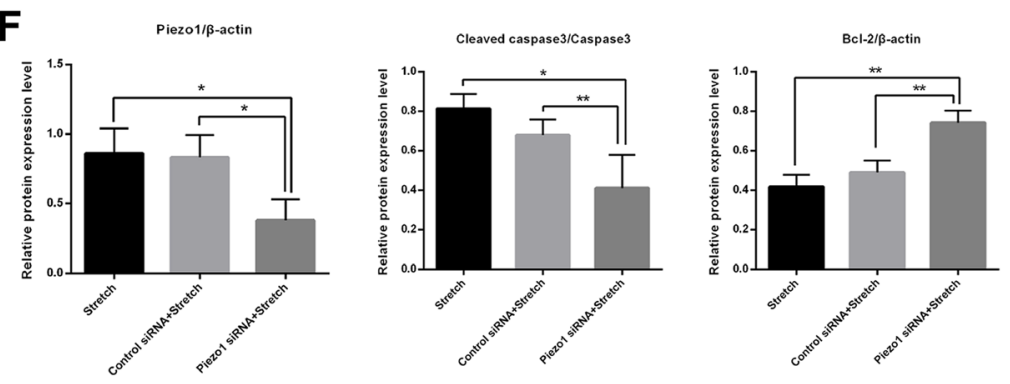

Fig. 2 (See legend on next page.) 


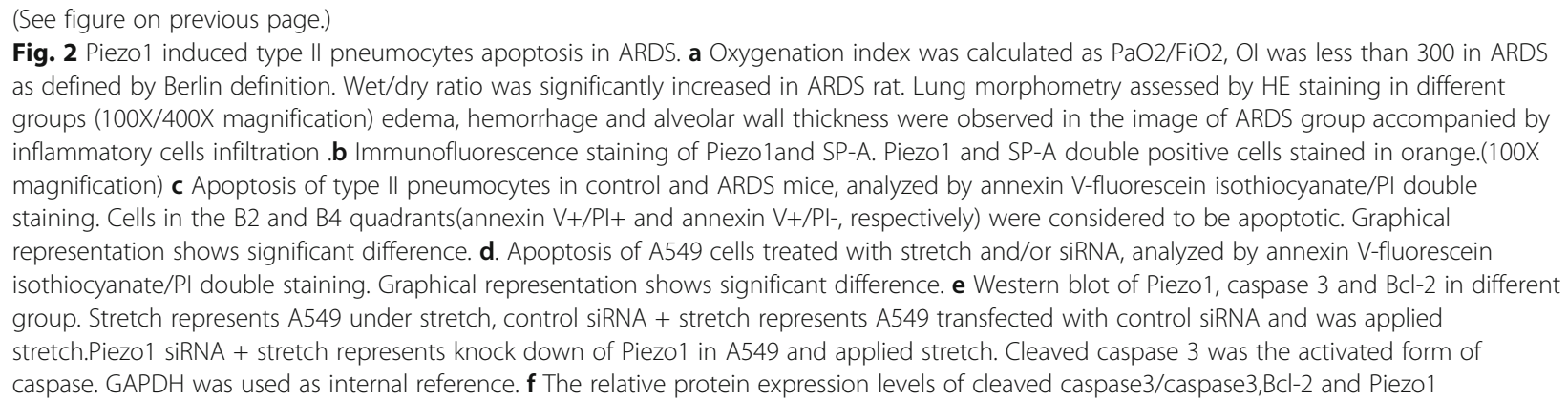

mainly expressed in type II pneumocytes, and numerous studies have provided evidence that apoptosis of type II pneumocytes is one of the mechanisms of ARDS, we sought to determine whether Piezo1 mediated type II pneumocytes apoptosis in ARDS. First, we isolated type II pneumocytes and confirmed by immunofluorescence staining of SP-C(Additional file 1: Figure S1B). Then we analyzed the apoptosis of type II pneumocytes from control and ARDS rats by Annexin V/PI staining with flow cytometry. Consistent with other studies, the apoptosis of type II pneumocytes was markedly increased in ARDS rats in our study(Fig. 2c).

To test whether this apoptosis was partly due to the upregulation of Piezo1, alveolar epithelial cells A549 were transfected with silent RNA to knock down Piezo1 expression. The efficacy of knockdown was assessed by qPCR(Additional file 1: Figure S2A). Because Piezo1 is a "stretch sensing" protein, as reported before, and one of the mechanisms of ARDS is stress-induced lung injury, we applied stretch to A549 cells to mimic the mechanical stretch between individual alveoli due to heterogeneous compliance in ARDS. The apoptosis induced by mechanical stretch was intensity-dependent and 15\% mechanical stretch could induce moderate apoptosis(Additional file 1: Figure S2B),thus, we used this intensity in the subsequent experiments. It was shown that knockdown of Piezo1 reduced stretch-induced apoptosis (Fig. 2d).

To further confirm that Piezo1 mediates apoptosis and determine the pathway involved, we performed western blot to assay the apoptosis-related proteins. Caspase-3 is activated in apoptotic cells both by extrinsic (death ligand) and intrinsic (mitochondrial) pathways and Bcl-2 is localized to the outer membrane of mitochondria and regulates intrinsic apoptosis. From western blot analysis, Piezo1 was successfully knocked down in A549, accompanied by decreased cleavage of caspase-3 (Fig. 2e, f) and increased Bcl-2 (Fig. 2e, f). These results demonstrated that Piezo1 mediated type II apoptosis of pneumocytes in ARDS through the Bcl-2 pathway.

\section{Piezo1 mediated apoptosis depends on calcium influx} Our observations indicated that Piezo1-mediated apoptosis was related to $\mathrm{Bcl}-2$. $\mathrm{Bcl}-2$ proteins were critical regulators of intracellular $\mathrm{Ca} 2+$ dynamics [21] whereby Piezo1 was a selected cation channel that controls stretch induced $\mathrm{Ca} 2+$ influx [22]. Therefore, we speculated that activated Piezo1 during stretch increased $\mathrm{Ca} 2+$ influx and induced apoptosis. To verify the this, we assayed $\mathrm{Ca} 2+$ influx by detecting the fluorescence intensity of $\mathrm{Ca} 2+$.Stretch increased intracellular $\mathrm{Ca} 2+$ concentration and this elevation was absent when $\mathrm{Ca} 2+$ was deprived in medium(Fig. 3a), demonstrating that the elevated $\mathrm{Ca} 2+$ was due to $\mathrm{Ca} 2+$ influx rather than $\mathrm{Ca} 2+$ release from storage. Notably, we observed that knockdown of Piezo1 dramatically reduced Ca2+ influx during stretch(Fig. 3b) The apoptosis was of no significant change between the cells with or without Piezo1 knockdown when $\mathrm{Ca} 2+$ was deprived(Fig. 3d, e) We next assessed the expression of caspase 3 and Bcl-2 of A549 in $\mathrm{Ca} 2+$ free medium. Expression of $\mathrm{Bcl}-2$ increased in $\mathrm{Ca} 2+$ free condition, indicating the negative relationship between $\mathrm{Ca} 2+$ and $\mathrm{Bcl}-2$ (Fig. 3d, e).

Piezo1 is a potential mechanism to explain the protective role in lung injury of low tidal volume ventilation

Low tidal volume ventilation(LTV) has been recommended as a lung protective strategy in ARDS. However, the mechanisms are not well clarified. LTV uses a low volume ventilation $(4-6 \mathrm{cmH} 2 \mathrm{O})$ with a proper PEEP to avoid lung overdistension and opens collapsed alveoli. This reduces ventilation homogeneity, and thus alleviates stretch forces in lung parenchyma at margins between aerated and atelectatic regions. Therefore, we examined whether Piezo1 explained part of this function of LTV by ventilating oleic acid injected rats with either LTV(Vt $6 \mathrm{cmH} 2 \mathrm{O} / \mathrm{kg}$, PEEP $3 \mathrm{cmH} 2 \mathrm{O}$ )or high tidal volume ventilation $\mathrm{HVT}(\mathrm{Vt} 15 \mathrm{cmH} 2 \mathrm{O} / \mathrm{kg}$, PEEP $0 \mathrm{cmH} 2 \mathrm{O})$ .Rats with LTV presented less severe ARDS and lung injury(Fig. 4a Additional file 1: Figure S3) In addition, the apoptosis of type II pneumocytes was significantly reduced in LTV rats compared with HVT rats(Fig. 4b) Notably, we observed less expression of Piezo1 in LTV rats. In line with the relationship between Piezo1 and $\mathrm{Ca} 2+$ influx we've revealed above, rats ventilated with low volume had reduced $\mathrm{Ca} 2+$ influx (Fig. 4d) and caspase 3 accompanied with elevated Bcl-2 (Fig. 4e)These 

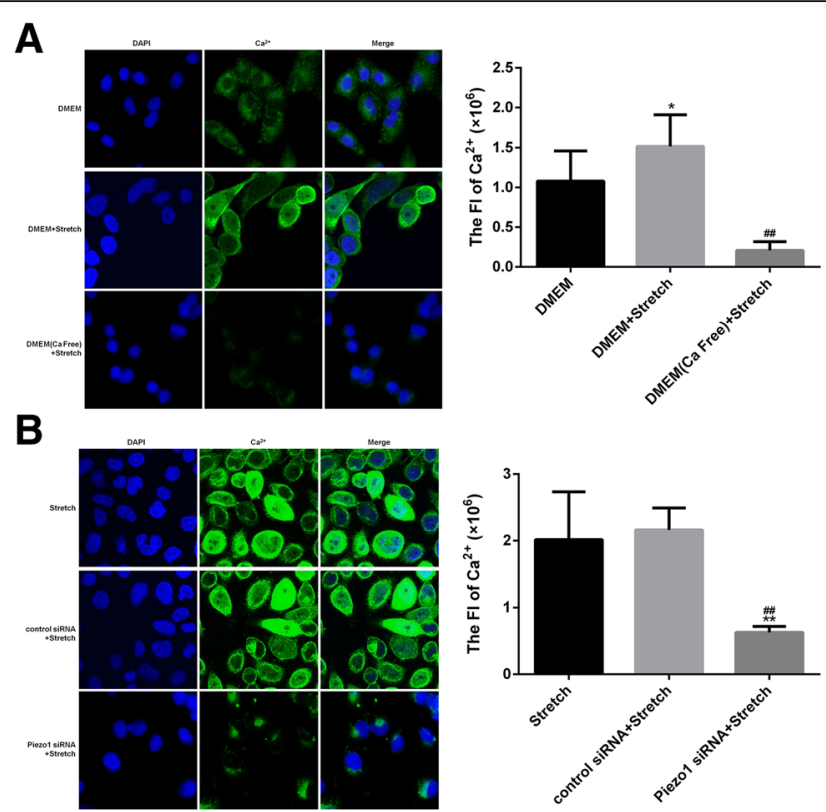

C
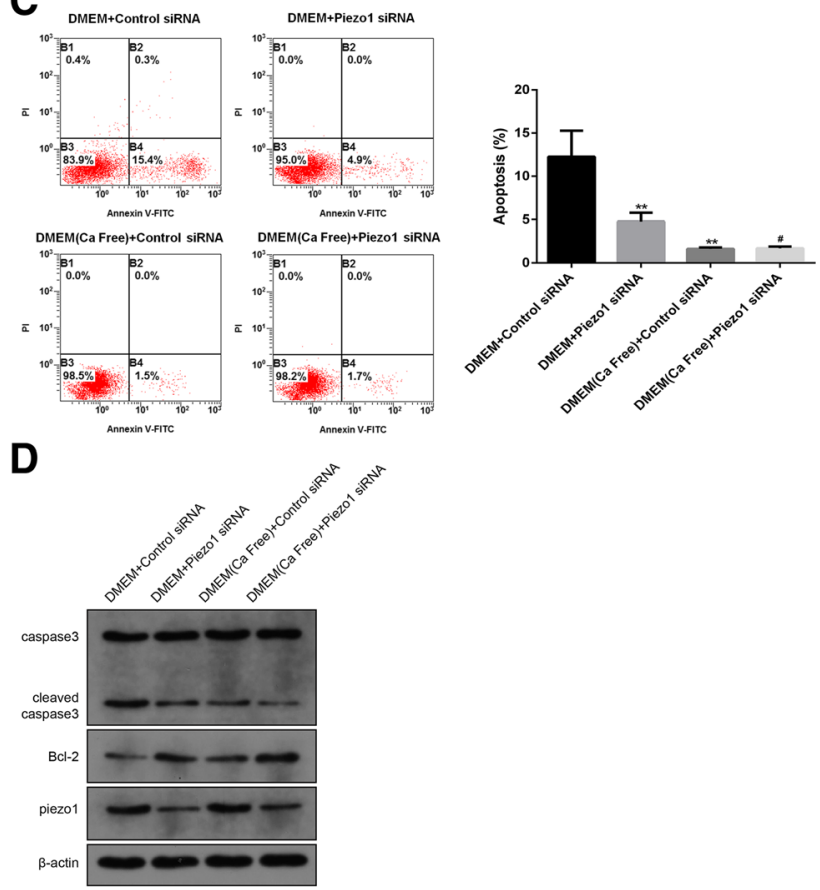

\section{E}
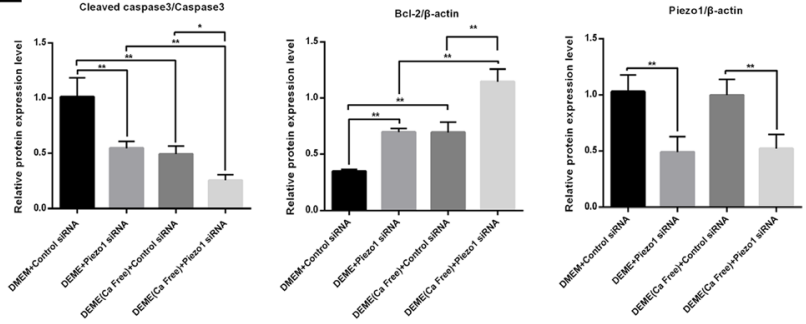

Fig. 3 (See legend on next page.) 
(See figure on previous page.)

Fig. 3 Piezo 1 mediated apoptosis depends on calcium influx. a A549 cells were treated with $15 \%$ stretch with a frequency of $0.5 \mathrm{HZ}$ for $4 \mathrm{~h}$. Ca2+ was labeled with10 $\mu \mathrm{M}$ Fluo-3/AM and observed by confocal imaging. Selected eight visual fields randomly and detected total fluorescent intensity by Image pro Plus 6.0.Calculatedsingle cell fluorescent intensity as total fluorescent intensity/cell counts. In Ca2+ free group, calcium was removed from medium. (1000 × magnification) b A549 was transfected with Piezo1-siRNA72hrsbefore treated with mechanical stretch and Ca2+ influx was assayed by confocal imaging. (1000 × magnification) c The apoptosis of A549 treated with mechanical stretch was assessed by flow cytometry and annexin V-fluorescein isothiocyanate/PI double staining. ${ }^{* *} p<0.01$.vs. DMEM +Control siRNA; \#\#p $<0.01$.vs. DMEM+Piezo1 siRNA. d The expression of apoptosis related proteins are assayed by western blot. e The relative protein expression levels of cleaved caspase3/ caspase3,BCl-2 and Piezo 1

results support that LTV-protected lungs may partly be due to the regulation of Piezo1.

\section{Discussion}

The mechanisms of ARDS have not been well clarified todate, and a lack of specific pharmacotherapies has lead to high mortality. Here for the very first time, we have found Piezo1, expressed in type II pneumocytes of rats which may be likely potential targets for treatment of ARDS. Furthermore, we demonstrated that Piezo1-mediated apoptosis of type II pneumocytes via increased calcium influx during ARDS. In addition, our data revealed a potential mechanism of low tidal volume ventilation in alleviating lung injury through regulating Piezo1 expression.

Alveolar epithelium injury contributes significantly to the development of acute pulmonary edema which eventually results in ARDS-related lung injury [23]. As one of the components of alveolar epithelia, type II pneumocytes have many functions, including surfactant production [24], immune function [25] as well as clearance of alveolar edema fluid [26] We observed that Piezo1 in type II pneumocytes was upregulated during ARDS, and our results as well as that of other published studies [27-30] indicated that apoptosis of type II pneumocytes increased and played a vital role in lung injury. Therefore, we assumed that Piezo1 mediated the apoptosis of type II pneumocytes. To verified this, we used mechanical stretch to establish an in vitro model since the inhomogeneous stress of alveoli in ARDS contributed a big part to the process, besides, as a mechanical ion channel sensing various types of mechanical stretch [6, 11, 31-35], Piezo1 was induced by the mechanical stretch. Consistent with our hypothesis, we found that knockdown of Piezo1 dramatically reduced the apoptosis of type II pneumocytes and we also found it was related to Bcl-2, the proteins that mediate the process by which mitochondria contribute to cell death known as the intrinsic apoptosis pathway [21] In line with our results, it was reported that stretch-induced apoptosis of type II pneumocytes was mediated by the intrinsic mitochondrial pathway rather than extrinsic pathway [36].

Furthermore, we found Piezo1 induced apoptosis in a calcium-dependent way.Piezo1 was new family of mechanosensitive ion channels that allow transmembrane $\mathrm{Ca} 2+$ flux into cells, they were associated with the physiological response to touch, pressure, and stretch [14]. Naohiko Murata et al. found mechanical stretch induced $\mathrm{Ca} 2+$ influx in human lung fibroblast, which was not caused by activation of conventional stretch-sensitive ion channels [37]. We demonstrated that Piezo1 knockdown reduced $\mathrm{Ca} 2+$ influx during stretch rather than $\mathrm{Ca} 2+$ release from internal $\mathrm{Ca} 2+$ storage. Furthermore, removal of $\mathrm{Ca} 2+$ diminished Piezo1 mediated apoptosis. These suggested that $\mathrm{Ca} 2+$ influx was likely to be a "second message" followed by activation of Piezo1 that signaled the apoptosis pathway. Ca2+ overload played a vital role in inducing apoptosis [38]. In our results, we found that Piezo1-induced apoptosis was related to Bcl2 , and the diminish of $\mathrm{Ca} 2+$ increased $\mathrm{Bcl}-2$ expression to a level comparable to Piezo1 knockdown. These results implies the apoptosis generated by Piezo1-induced $\mathrm{Ca} 2+$ influx may be associated with $\mathrm{Bcl}-2$ change. It was unveiled that $\mathrm{Bcl}-2$ regulated calcium homeostasis and protected cells from apoptosis by reducing $\mathrm{Ca} 2+$ overload [21]. Therefore, Bcl-2 may involve in Piezo1-induced apoptosis by functioning as a calcium regulated protein. Christopher J. Winters et.al revealed that CAMKII inhibition blunted bleomycin-induced apoptosis in type II pneumocytes by decreasing cytoplasmic $\mathrm{Ca} 2+$ concentration, the potential mechanism related to endoplasmic reticulum (ER) $\mathrm{Ca} 2+$ release. $\mathrm{Bcl}-2$ was demonstrated to reduce $\mathrm{Ca} 2+$ by competing for the ligand-binding of $\mathrm{Ca} 2+$ channel in ER [39]. Thus it was possible that CAMKII promotes the signaling pathway of $\mathrm{Ca} 2+-$ induced apoptosis whereas $\mathrm{Bcl}-2$ inhibits this process.

Patients with ARDS tend to have rapid and shallow breathing patterns due to a decreased lung volume, this promotes more intensified stress due to the increase of cyclic alveolar collapse and reopening, leading to upregulation of Piezo1.Mechanical ventilation is a common supportive therapy of ARDS, which relieves dyspnea and improves oxygen in patients. However, mechanical ventilation has also been shown to significantly contribute to aggravating lung injury, called ventilation induced lung injury(VILI),especially when used with inappropriately high tidal volumes [26]. Therefore, low tidal volume ventilation was recommended as a lung protective strategy to avoid alveolar overdistention. We compared LTV and HVT in our rat model and found that Piezo1 expression along with apoptosis and 


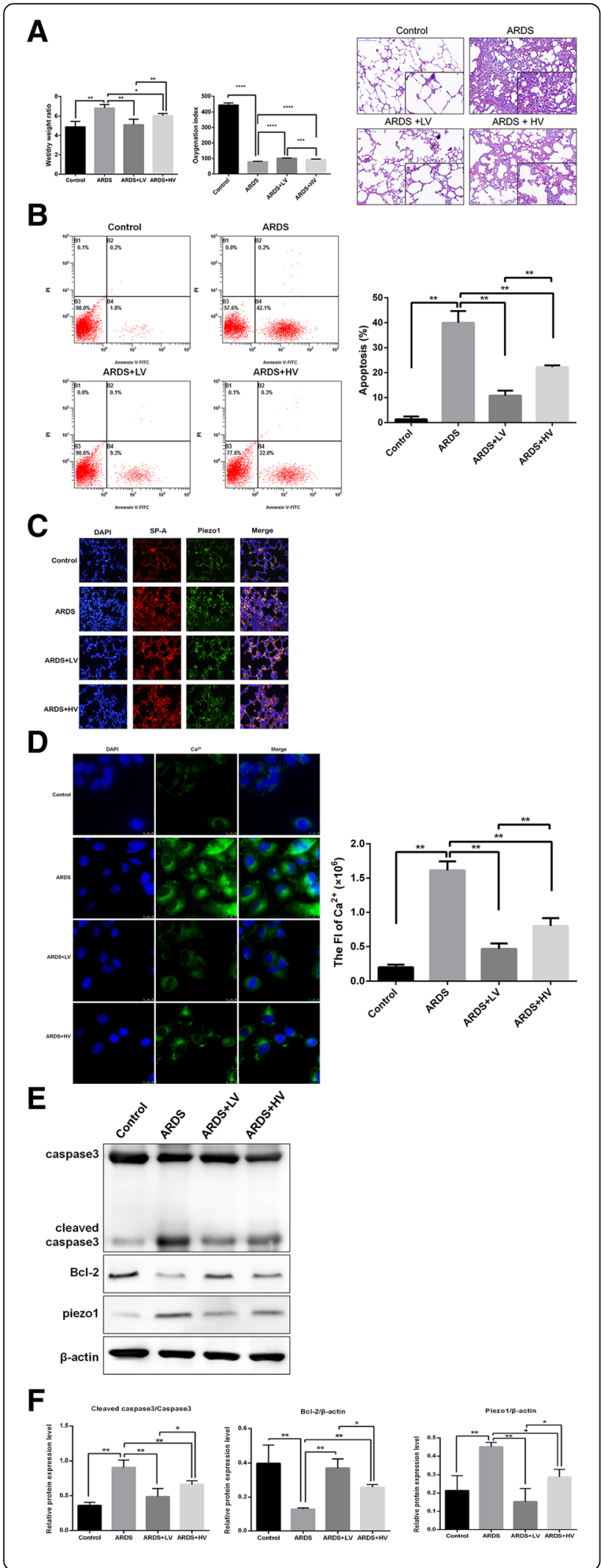

Fig. 4 Piezo 1 was a potential mechanism to explain the protective role in lung injury of LTV. a Wet/dry ratio decreased and oxygenation index (calculated as $\mathrm{PaO} 2 / \mathrm{FiO} 2$ ) in different group. Lung morphometry assessed by HE staining (100X/400X magnification) $\mathbf{b}$ The apoptosis of type II pneumocytes assayed by flow cytometry. c Immunofluorescence staining of Piezo1 and SP-A. Piezo 1 and SP-A double positive cells stained in orange.(100 $x$ magnification) $\mathbf{d}$ Ca2+ influx detected by confocal microscope and the quantitation of immunofluorescence presented as a bar graph. (1000 $\times$ magnification) e The expression of caspase 3,BCl-2 and Piezo 1 in rats assessed by western blot (cleaved caspase 3 was an activated form of caspase 3 ) (NS, not significant. *, $p<0.05 .{ }^{* *}, p<$ 0.001.LV low tidal volume, HV high tidal volume.) $f$ The relative protein expression levels of cleaved caspase3/caspase3,Bcl-2 and Piezo 1

$\mathrm{Ca} 2+$ influx was significantly reduced when low tidal volume was used. This supports that Piezo1 may be a potential mechanism to explain the protective role of LTV.

\section{Conclusions}

The results of our studies demonstrated that Piezo1 played an pivotal role in lung injury. Activation of Piezo1 by mechanical stretch induced the apoptosis of type II pneumocytes via $\mathrm{Ca} 2+$ influx. Inhibition of Piezo1 or $\mathrm{Ca} 2+$ influx may become a potential target for preventive or therapeutic interventions aiming at relieving lung injury in ARDS. The Piezo1 mediated apoptosis was related to Bcl-2, but further research is required to support this.

\section{Additional file}

Additional file 1: Figure S1. The expression of Piezo1 on rat type $\|$ pneumocytes. A)The level of IL-1 $\beta$ and TNF-a increased in ARDS rats, tested by ELISA assay. B)Identification of Type II pneumocytes isolated from lung. Nuclei stained with DAPI, Positive staining depicted in blue. SP-C containing cells stained in green. SP-C is a specific marker for type II pneumocytes $(1000 \times$ magnification). Figure S2. Piezo1 induced type II pneumocytes apoptosis in ARDS.A)The efficacy of knockdown of piezo1 was evaluated by Q-PCR and siRNA-1 was used in the following experiments. B) The apoptosis of A549 cells strength of mechanical stretch, assayed by annexin $\mathrm{V}$-fluorescein isothiocyanate/PI double staining. Figure S3. Piezo1 was a potential mechanism to explain the protective role in lung injury of LTV. The level of IL-1ßand TNF-a in different groups, tested by ELISA assay. (DOCX $605 \mathrm{~kb}$ )

\section{Abbreviations}

ARDS: acute respiratory distress syndrome; HE: hematoxylin and eosin; IL$1 \beta$ : interleukins-1 $\beta$; MV: mechanical ventilation; PEEP: positive end-expiratory pressure; SD: Sprague-Dawley; SP-A: pulmonary surfactant protein A; TNFa: tumor necrosis factor-a; W/D: wet-to-dry ratio

\section{Acknowledgements}

Not applicable.

\section{Authors' contributions}

G-PL and JX conceived, designed and performed the experiments, drafted the manuscript; $L-L C$ assisted in parts of the experiments; $L-L C$ and $Y-H Z$ helped analyze the statistics; YK,B-XC, JY helped in the draft of the article; KY and Z-ZW designed the experiments and revised the article. All authors read and approved the final manuscript. 


\section{Funding}

This study was supported by National Natural Science Foundation of China (31301116); Project of Sichuan Provincial Education Department(11ZA297); Project of Sichuan Science and Technology(2019YFS0235).

\section{Availability of data and materials}

The datasets used and/or analyzed during the current study are available from the corresponding author on reasonable request.

\section{Ethics approval and consent to participate}

The protocol was approved by the Institutional Animal Care and Treatment Committee of Sichuan University (Chengdu, P.R. China).

\section{Consent for publication}

Not applicable.

\section{Competing interests}

"Author Li-li Cao" is currently receiving a grant (31301116) from National Natural Science Foundation of China and "Author Z. W Zhang" is currently receiving a grant(11ZA297) from Education Department of Sichuan Province (CN). "Author GuoPeng Liang "is currently receiving a grant(2019YFS0235) from Project of Sichuan Science and Technology.The remaining authors have disclosed that they do not have any conflicts of interest.

\section{Publisher's Note}

Springer Nature remains neutral with regard to jurisdictional claims in published maps and institutional affiliations.

\section{Author details}

'Department of Critical Care Medicine, West China School of Medicine and West China Hospital, Sichuan University, Chengdu 610041, China. ${ }^{2}$ Chengdu Medical College, Chengdu 610041, Sichuan, China. ${ }^{3}$ Department of Respiratory Critical Care Medicine, West China School of Medicine and West China Hospital, Sichuan University, Chengdu 610041, China.

Received: 8 March 2019 Accepted: 24 May 2019

Published online: 11 June 2019

\section{References}

1. Bellani G, Laffey JG, Pham T, et al. Epidemiology, patterns of care, and mortality for patients with acute respiratory distress syndrome in intensive care units in 50 countries. JAMA. 2016;315(8):788-800.

2. Force ADT, Ranieri VM, Rubenfeld GD, et al. Acute respiratory distress syndrome: the Berlin definition. JAMA. 307(23):2526-33.

3. Sawa T. The molecular mechanism of acute lung injury caused by Pseudomonas aeruginosa: from bacterial pathogenesis to host response. J Intensive Care. 2014;2(1):10.

4. Coste B, Mathur J, Schmidt M, et al. Piezo1 and Piezo2 are essential components of distinct mechanically activated cation channels. Science. Oct 01 2010:330(6000):55-60.

5. Syeda R, Florendo MN, Cox CD, et al. Piezol channels are inherently mechanosensitive. Cell Rep. 2016:17(7):1739-46.

6. Cahalan SM, Lukacs V, Ranade SS, Chien S, Bandell M, Patapoutian A. Piezo1 links mechanical forces to red blood cell volume. Elife. May 22 2015:4.

7. Li J, Hou B, Tumova S, et al. Piezol integration of vascular architecture with physiological force. Nature. Nov 13 2014:515(7526):279-82.

8. Faucherre A, Kissa K, Nargeot J, Mangoni ME, Jopling C. Piezo1 plays a role in erythrocyte volume homeostasis. Haematologica. Jan 2014;99(1):70-5.

9. Ilkan Z, Wright JR, Goodall AH, Gibbins JM, Jones Cl, Mahaut-Smith MP. Evidence for shear-mediated Ca2+ entry through mechanosensitive cation channels in human platelets and a megakaryocytic cell line. J Biol Chem. Apr 172017

10. Ranade SS, Qiu Z, Woo SH, et al. Piezo1, a mechanically activated ion channel, is required for vascular development in mice. Proc Natl Acad Sci U S A. 2014:111(28):10347-52.

11. Retailleau K, Duprat F, Arhatte M, et al. Piezo 1 in smooth muscle cells is involved in Hypertension-dependent arterial remodeling. Cell Rep. Nov 10 2015;13(6):1161-71.
12. Wang S, Chennupati R, Kaur H, Iring A, Wettschureck N, Offermanns S Endothelial cation channel PIEZO1 controls blood pressure by mediating flow-induced ATP release. J Clin Invest. Dec 01 2016;126(12):4527-36.

13. Hypertension ASJ. Mechanosensation by PIEZO1 in blood pressure control. Nat Rev Nephrol. 2017;13(1):3.

14. Miyamoto $T$, Mochizuki $T$, Nakagomi $H$, et al. Functional role for Piezo1 in stretch-evoked $\mathrm{ca}(2)(+)$ influx and ATP release in urothelial cell cultures. J Biol Chem. 2014;289(23):16565-75.

15. Bagriantsev SN, Gracheva EO, Gallagher PG. Piezo proteins: regulators of Mechanosensation and other cellular processes. J Biol Chem. Nov 14 2014:289(46):31673-81.

16. Chiumello D, Carlesso E, Cadringher $P$, et al. Lung stress and strain during mechanical ventilation for acute respiratory distress syndrome. Am J Respir Crit Care Med. Aug 15 2008;178(4):346-55.

17. Oba Y, Salzman GA. Ventilation with lower tidal volumes as compared with traditional tidal volumes for acute lung injury. $N$ Engl J Med. 2000:343(11):813 author reply 813-814.

18. Caironi P, Cressoni M, Chiumello D, et al. Lung opening and closing during ventilation of acute respiratory distress syndrome. Am J Respir Crit Care Med. Mar 15 2010;181(6):578-86.

19. Wang $Y Y$, Zhang $H$, Ma $T$, et al. Piezol mediates neuron oxygenglucose deprivation/reoxygenation injury via ca/calpain signaling. Biochem Biophys Res Commun. 2019;513(1):147-53.

20. Belinsky SA, Lechner JF, Johnson NF. An improved method for the isolation of type II and Clara cells from mice. In Vitro Cell Dev Biol Anim. May 1995;31(5):361-6.

21. Hardwick JM, Soane L. Multiple functions of BCL-2 family proteins Cold Spring Harb Perspect Biol. Feb 1 2013:5(2).

22. Cinar E, Zhou S, DeCourcey J, Wang Y, Waugh RE, Wan J. Piezol regulates mechanotransductive release of ATP from human RBCs. Proc Natl Acad Sci U S A. Sep 22 2015;112(38):11783-8.

23. Matthay MA, Zemans RL. The acute respiratory distress syndrome: pathogenesis and treatment. Annu Rev Pathol. 2011;6:147-63.

24. Wright JR, Hawgood S. Pulmonary surfactant metabolism. Clin Chest Med. 1989;10(1):83-93

25. Harbeck RJ, Gegen NW, Struhar D, Mason R. Class II molecules on rat alveolar type II epithelial cells. Cell Immunol. 1988;111(1):139-47.

26. Gill SE, Yamashita CM, Veldhuizen RA. Lung remodeling associated with recovery from acute lung injury. Cell Tissue Res. 2017;367(3):495-509.

27. Steinberg $H$, Greenwald RA, Sciubba J, Das DK. The effect of oxygenderived free radicals on pulmonary endothelial cell function in the isolated perfused rat lung. Exp Lung Res. 1982;3(2):163-73.

28. Li X, Shu R, Filippatos G, Uhal BD. Apoptosis in lung injury and remodeling. J Appl Physiol (1985). 2004;97(4):1535-42.

29. Galani V Tatsaki $E$, Bai $M$, et al. The role of apoptosis in the pathophysiology of acute respiratory distress syndrome (ARDS): an up-to-date cell-specific review. Pathol Res Pract. Mar 15 2010;206(3): 145-50.

30. Song Y, Mao B, Qian G. The role of apoptosis and Fas/FasL in lung tissue in patients with acute respiratory distress syndrome. Zhonghua Jie He He Hu Xi Za Zhi. Oct 1999;22(10):610-2.

31. Martins JR, Penton D, Peyronnet $R$, et al. Piezo1-dependent regulation of urinary osmolarity. Pflugers Arch. Jul 2016:468(7):1197-206.

32. Wright JR, Amisten S, Goodall AH, Mahaut-Smith MP. Transcriptomic analysis of the ion channelome of human platelets and megakaryocytic cell lines. Thromb Haemost. Aug 01 2016;116(2):272-84.

33. Peyronnet R, Martins JR, Duprat F, et al. Piezo1-dependent stretch-activated channels are inhibited by Polycystin-2 in renal tubular epithelial cells. EMBO Rep. Dec 2013;14(12):1143-8.

34. Michishita M, Yano K, Tomita Kl, Matsuzaki O, Kasahara Kl. Piezol expression increases in rat bladder after partial bladder outlet obstruction. Life Sci. Dec 01 2016;166:1-7

35. Miyamoto T, Nakagomi H, Kira S, et al. Piezo1, a novel Mechanosensor in the bladder Urothelium. Neurourol Urodynam. 2012;31(6):1015-7.

36. Kuhn H, Nieuwenhuiisen $H$, Karthe B, Wirtz H. Stretch-induced apoptosis in rat alveolar epithelial cells is mediated by the intrinsic mitochondrial pathway. Experimental lung research. 2017:43(1):49-56.

37. Murata N, Ito S, Furuya K, et al. Ca2+ influx and ATP release mediated by mechanical stretch in human lung fibroblasts. Biochem Bioph Res Co. 2014 453(1):101-5 
38. Rong YP, Aromolaran AS, Bultynck G, et al. Targeting BCl-2-IP3 receptor interaction to reverse $\mathrm{BCl}-2$ 's inhibition of apoptotic calcium signals. Mol Cell. Jul 25 2008;31(2):255-65.

39. Ivanova $H$, Wagner $L E$, Tanimura A, et al. BCl-2 and IP compete for the

ligand-binding domain of IPRs modulating ca signaling output. Cellular and molecular life sciences : CMLS. 2019.

Ready to submit your research? Choose BMC and benefit from:

- fast, convenient online submission

- thorough peer review by experienced researchers in your field

- rapid publication on acceptance

- support for research data, including large and complex data types

- gold Open Access which fosters wider collaboration and increased citations

- maximum visibility for your research: over $100 \mathrm{M}$ website views per year

At $B M C$, research is always in progress.

Learn more biomedcentral.com/submissions 